\title{
Distribuição do macrozoobentos na coluna vertical dos sedimentos da região estuarina de Cananéia (SP), Brasil
}

\author{
(Vertical distribution of macrobenthic animals in bottom sediments in the estuarine \\ region of Cananéia, southern Brazil)
}

\author{
Adriana Jorcin \\ Instituto de Biociências, UNESP \\ Departamento de Zoologia \\ (Campus de Botucatu, Rubião Junior, 18618-000, Botucatu, SP, Brasil)
}

$\mathrm{O}$ ambiente estuarino encontra-se dominado por sedimentos de granulometria fina, os quais são introduzidos no estuário tanto pelo oceano como pelos rios. Estes sedimentos são ricos em partículas alimentares, porém pobres em oxigênio e com freqüência anóxicos.

Os organismos bentônicos desempenham um significativo papel no intercâmbio de nutrientes, gases dissolvidos e outros materiais entre 0 sedimento e a interface água-sedimento (Nalepa \& Robertson, 1981). A variação na distribuição dos organismos na coluna de sedimentos pode ser explicada em função da variação de alguns fatores físicos e químicos, sendo a penetração de oxigênio dentro do substrato uma das principais variáveis a ser considerada (Kirchner, 1975). Vários autores têm estudado a relação entre a distribuição da fauna bêntica na coluna vertical de sedimentos $\mathrm{e}$ as características físicas e químicas dos mesmos (Flint \& Kalke, 1986; Dauer et al., 1987; Nilsson \& Rosenberg, 1994), assim como o efeito das atividades dos organismos na modificação de tais características (Rhoads \& Young, 1970; Rhoads, 1974; Duplisea \& Hargrave, 1996; Flach \& Heip, 1996).

O objetivo deste estudo foi determinar a distribuição vertical dos organismos macrobentônicos na coluna de sedimentos da região estuarina de Cananéia (SP), e sua relação com as características granulométricas e potencial de óxidoredução no perfil vertical.

A região estuarina-lagunar de Cananéia localiza-se no litoral sul do Estado de São Paulo $\left(25^{\circ} \mathrm{S}, 45^{\circ} \mathrm{W}\right)$, formada por um sistema de canais conectados com o mar por duas entradas (Barra de Cananéia e Barra de lguape). A área ocupa uma extensão de $110 \mathrm{~km}^{2} \mathrm{e}$ a circulação de água nos canais é dominada pelo regime de maré e pelos aportes de água doce, principalmente durante os períodos de chuva. A salinidade é controlada pela intrusão das marés no sistema, sendo a mesma mais alta na Baía de Trapandé (Schaeffer-Novelli et al., 1990).

Para a realização do trabalho coletou-se as amostras a cada 3 meses (13/14 fevereiro, 03/04 maio; 28/29 agosto, 08/09 novembro) durante o ano de 1995. Em cada período de amostragem 3 "corers" $(6,5 \mathrm{~cm}$ de diâmetro interno) foram enterrados manualmente no sedimento em 8 estações de coleta (Fig. 1). Para determinar a distribuição vertical dos invertebrados nas colunas de sedimentos obtidas através dos "corers", estas foram cortadas nas seguintes profundidades: $0-1$, $1-2,2-5,5-10-10-15$ e $15-20 \mathrm{~cm}$. Após seccionadas as amostras foram fixadas com formol $8 \%$. Os invertebrados foram separados do sedimento com uma malha de $500 \mu \mathrm{m}$ identificados até o menor nível taxonômico possível, e conservados em álcool $70 \%$. A densidade de organismos foi expressa em $300 \mathrm{~cm}^{3}$, para manter o volume de amostragem. Um quarto "corer" de sedimento foi coletado em cada estação de amostragem para medir o potencial de óxido-redução (Eh) (metodologia descrita por Zobell, 1946 e Haynes et al., 1958) e realizar as análises sedimentológicas (porcentagem de água e matéria orgânica, e diâmetro médio de grão) (Håkanson \& Jansson, 1983), nas mesmas profundidades mencionadas anteriormente. A concentração de matéria orgânica (MO) foi determinada pela perda de peso por ignição a $550^{\circ} \mathrm{C}$ durante $2 \mathrm{~h}$. 


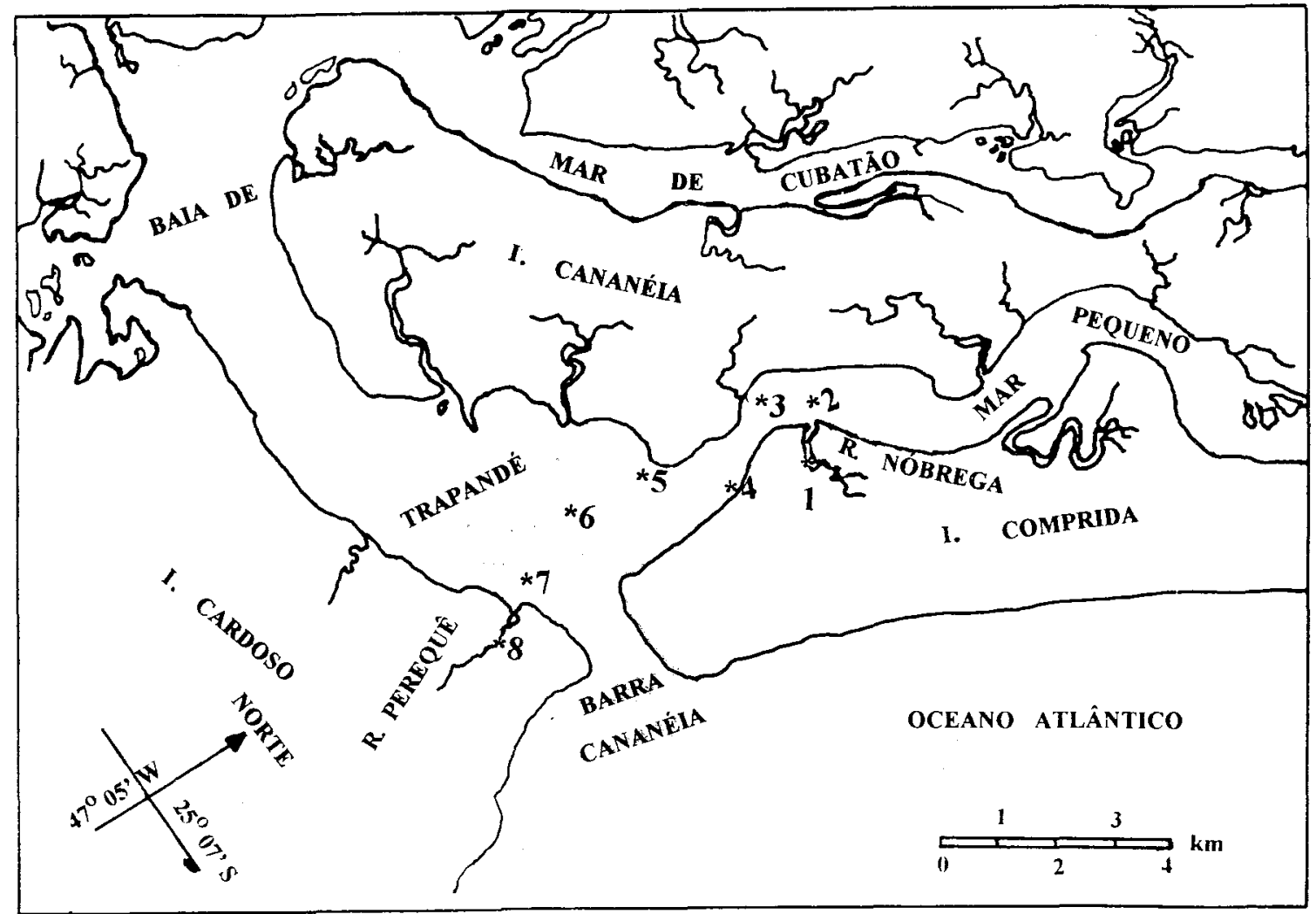

Fig. 1. Região estuarina de Cananéia, SP. Localização das estações de amostragem.

\section{Características dos sedimentos}

O predomínio de areia fina foi observado em praticamente todas as estações de amostragem, nos distintos estratos da coluna vertical do sedimento. Somente na estação 3 observou-se a presença de areia grossa e muito grossa nas diferentes profundidades analisadas. As estações 1, 2 e 4 apresentaram as maiores concentrações de MO nos sedimentos, enquanto que a maior porcentagem de água foi registrada na estação 2 . Em geral observou-se uma diminuição da porcentagem de $\mathrm{MO}$ e água com o aumento da profundidade na coluna de sedimento (Fig. 2).

Os valores de Eh oscilaram amplamente entre as diferentes estações de amostragem bem como entre os períodos de coleta. A passagem entre sedimentos oxidados e sedimentos reduzidos (camada de descontinuidade do potencial de óxido-redução, RPD), ocorreu a diferentes profundidades, dependendo da estạção de amostragem considerada (Fig. 3).

\section{Composição e distribuição vertical da fauna macrobentônica}

A fauna macrobentônica da região estuarina de Cananéia foi representada por organismos pertencentes aos Crustácea, Mollusca, Annelida, Echinodermata, Sipunculida, Nematoda, Nemertinea, Aracnida e Insecta. $\mathrm{O}$ grupo mais representativo foi o dos poliquetos, com presença de espécies em todas as estações de amostragem. Por outro lado a ocorrência da Classe Insecta assim como de oligoquetos da família Naididae esteve restrita à estação 8 (Tab. 1). Dentre os crustáceos, o principal representante foi o tanaidáceo Kalliapseudes shubartii, e dentre os moluscos o bivalve Tagelus sp. Ambos ocorreram em todas as estações de amostragem, com exceção da estação 8 .

$\mathrm{Na}$ estação 6 um maior número de organismos alcançou maiores profundidades de enterramento. Os crustáceos penetraram até a profundidade de $15 \mathrm{~cm}$, enquanto que os moluscos e os poliquetos penetraram até $20 \mathrm{~cm}$ de profundidade (Fig. 3).

De um modo geral a densidade máxima de organismos foi observada entre o sedimento subsuperficial e os $5 \mathrm{~cm}$ de profundidade na coluna vertical (Fig. 4).

Vários autores fazem referência a importância da distribuição dos organismos bentônicos em relação as características dos sedimentos, principalmente ao conteúdo orgânico (Dauer et al., 1987) e potencial de óxido-redução (Grizzle \& Penniman, 1991). 

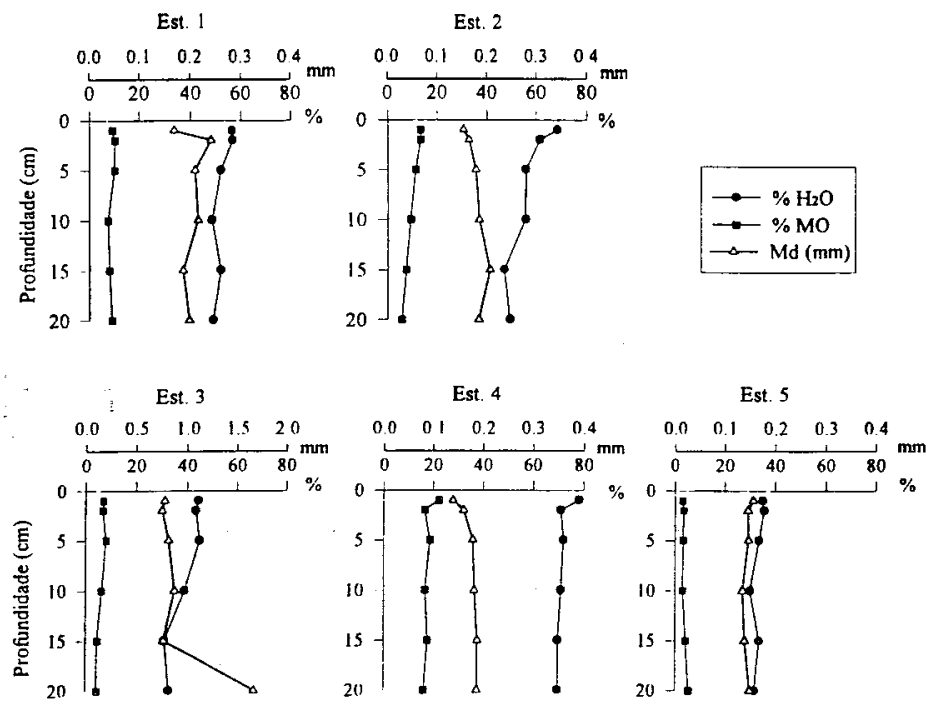

Est 6

Est 7

Est. 8

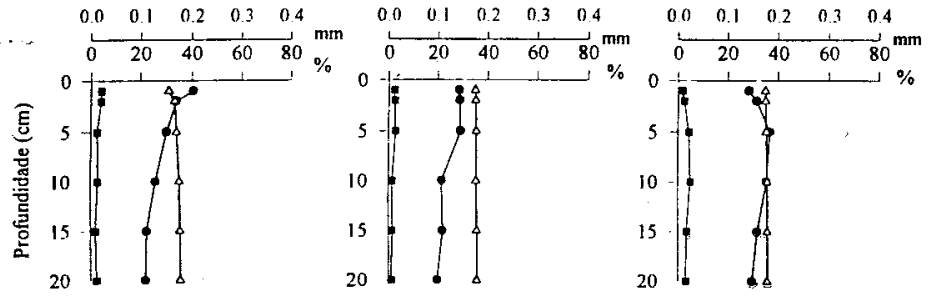

Fig. 2. Perfil vertical da concentração de água e matéria orgânica (\%) e do diâmetro médio do sedimento $(\mathrm{Md}, \mathrm{mm})$ nas estações de amostragem da região estuarina de Cananéia (SP).
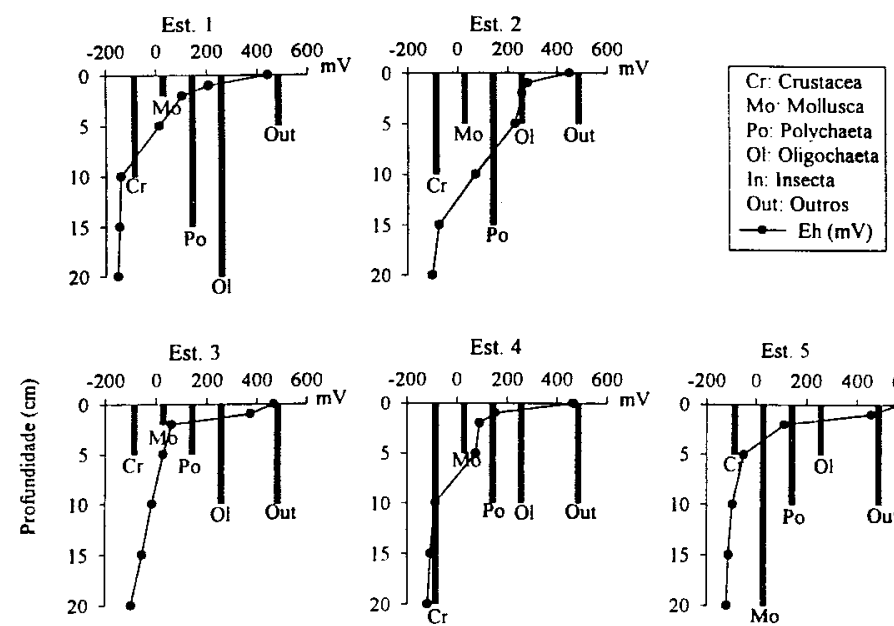

Est 5
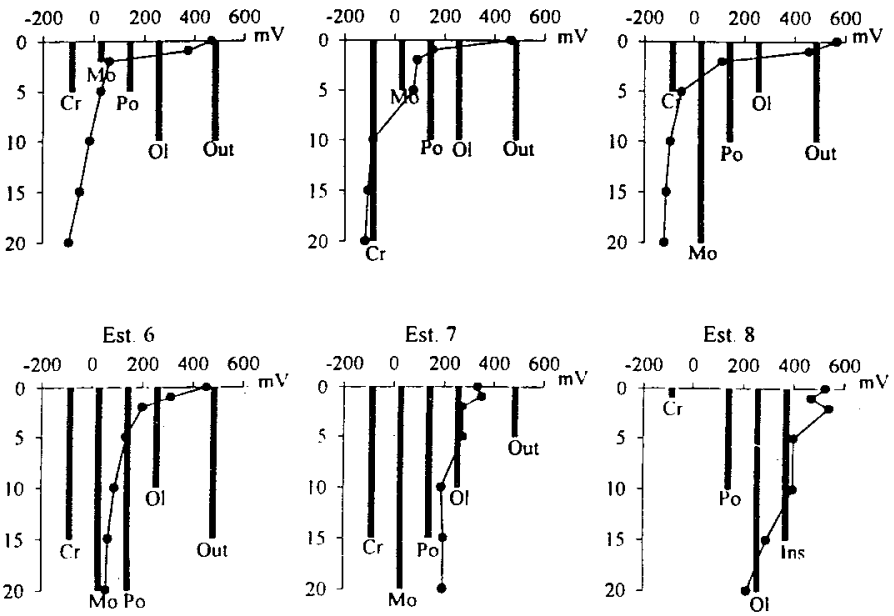

Fig. 3. Distribuição vertical do macrozoobentos na coluna de sedimentos, agrupado em grandes grupos, e perfil vertical do potencial de óxido-redução (Eh, $\mathrm{mV}$ ) nas estações de amostragem da região estuarina de Cananéia (SP). 
Tabela 1. Táxons encontrados nas estações de amostragem durante o período em estudo, na região estuarina de Cananéia (SP). * indica presença; - indica ausência.

\begin{tabular}{|c|c|c|c|c|c|c|c|c|}
\hline Taxon/Estação & 1 & 2 & 3 & 4 & 5 & 6 & 7 & 8 \\
\hline \multicolumn{9}{|l|}{ Crustacea } \\
\hline Goneplacidae & - & - & - & - & $*$ & - & - & - \\
\hline Clibanarius vittatus & - & - & - & - & - & - & $*$ & - \\
\hline Uca $\mathrm{sp}$ & - & * & - & $*$ & - & - & * & - \\
\hline Kalliapseudes shubartii & * & * & * & * & $*$ & $*$ & * & - \\
\hline Tanaidacea s/i & - & - & - & - & - & $*$ & * & - \\
\hline Tanais stanfordi & * & - & - & - & - & - & - & * \\
\hline Gammaridae & - & $*$ & - & - & - & - & - & - \\
\hline Flabellifera & - & $*$ & * & - & - & $*$ & - & - \\
\hline Cyprideis salebrosa & - & $*$ & - & * & $*$ & $*$ & - & - \\
\hline Cyprideis multidentata & - & - & - & $*$ & - & $*$ & - & - \\
\hline Cephalocarida & - & - & - & - & - & * & - & - \\
\hline Cumacea & $=$ & - & - & - & - & ${ }^{*}$ & - & - \\
\hline \multicolumn{9}{|l|}{ Mollusca } \\
\hline Tagelus $\mathrm{sp}$ & * & $*$ & * & * & $*$ & * & $*$ & - \\
\hline Macoma sp & $*$ & - & * & - & * & $*$ & * & - \\
\hline Thracia sp? & - & - & - & - & - & $*$ & - & - \\
\hline Anomalocardia brasiliana? & - & - & - & - & - & * & - & - \\
\hline Diplodonta $\mathrm{sp}$ & - & - & - & - & - & * & - & - \\
\hline Divaricella quadrisulcata & - & - & - & - & - & $*$ & - & - \\
\hline Lamellibranchia s/i & - & - & - & - & - & $*$ & * & - \\
\hline Heleobia $\mathrm{sp}$ ? & - & $*$ & - & $*$ & $*$ & - & - & - \\
\hline Olividae & - & $*$ & - & $*$ & * & $*$ & - & - \\
\hline \multicolumn{9}{|l|}{ Echinodermata } \\
\hline Ophiuroidea & - & - & - & - & - & $*$ & - & - \\
\hline Sipunculida & - & $*$ & $*$ & * & - & - & - & - \\
\hline Nematoda & * & $*$ & $*$ & $*$ & $*$ & $*$ & - & - \\
\hline Nemertinea & - & - & - & * & - & - & - & - \\
\hline \multicolumn{9}{|l|}{ Aracnida } \\
\hline Acarina & - & * & - & - & - & - & $*$ & - \\
\hline \multicolumn{9}{|l|}{ Insecta } \\
\hline Hydrophilidae & - & - & - & - & - & - & - & $*$ \\
\hline Ceratopogonidae & - & - & - & - & - & - & - & * \\
\hline Polypedilum sp. & - & - & - & - & - & - & - & $*$ \\
\hline Tripodura sp. & - & - & - & - & - & - & - & $*$ \\
\hline Tanylarsini Gen. $D(\#)$ & - & - & - & - & - & - & - & $*$ \\
\hline Tanytarsus sp. & - & - & - & - & - & - & - & * \\
\hline Goeldochironomus sp. & - & - & - & - & - & - & - & $*$ \\
\hline Rheotanytarsus sp. & - & - & - & - & - & - & - & $*$ \\
\hline
\end{tabular}


Tabela 1. (Cont.)

\begin{tabular}{|c|c|c|c|c|c|c|c|c|}
\hline Annelida & & & & & & & & \\
\hline Polychaeta & & & & & & & & \\
\hline Spiophanes wigleyi & * & * & * & $\bar{*}$ & - & * & - & - \\
\hline Prionospio heterobranchia & - & * & - & * & * & * & - & - \\
\hline Malacoceros vanderhorsti & - & - & $*$ & * & - & * & - & - \\
\hline Polydora sp. & - & - & * & - & - & - & * & - \\
\hline Spionidae s/I & * & $*$ & $*$ & * & * & * & $*$ & - \\
\hline Sigambra sp. & $*$ & $*$ & * & * & * & - & * & - \\
\hline Pilargis sp. & - & - & - & - & - & * & - & - \\
\hline Capitellidae & $*$ & * & $*$ & * & * & * & * & - \\
\hline Aricidea (acmira) cerrutii & - & * & - & * & * & * & * & - \\
\hline Glycera sp. & $*$ & * & - & * & * & * & - & - \\
\hline Gonianidae & - & $*$ & - & - & - & - & - & - \\
\hline Terebellides $\mathrm{sp}$. & - & - & * & - & - & - & - & - \\
\hline Exogone occidentalis & - & - & - & - & $*$ & * & - & - \\
\hline Lumbrineris sp. & - & * & * & - & - & * & - & - \\
\hline Ninoe sp. & - & $*$ & * & - & - & - & - & - \\
\hline Apistobranchus sp. & - & - & - & - & - & - & * & - \\
\hline Magelona sp. & - & - & - & - & - & * & - & - \\
\hline Poecilochaetus sp. & - & - & - & - & - & * & - & - \\
\hline Nephtys sp. & - & - & - & - & - & - & * & $*$ \\
\hline Galatowenia sp. & - & - & - & - & - & * & - & - \\
\hline Owenia $\mathrm{sp}$. & - & - & $*$ & - & - & - & - & - \\
\hline Armandia sp. & - & - & - & - & - & * & - & - \\
\hline Maldanidae & - & $*$ & - & - & * & * & - & - \\
\hline Ctenodrilidae & - & - & - & - & $*$ & - & - & - \\
\hline Sabellidae & - & $*$ & - & - & $*$ & - & - & - \\
\hline Nothria sp. & - & $*$ & - & - & - & - & - & - \\
\hline Hyalinoecia sp. & - & - & - & - & $*$ & - & - & - \\
\hline Leanira sp. & - & $*$ & - & - & - & - & - & - \\
\hline Pholoe sp. & - & $*$ & - & - & - & * & - & - \\
\hline Eumida sp. & - & $*$ & - & - & $*$ & - & - & - \\
\hline Leodamas sp. & - & - & - & - & - & - & * & - \\
\hline Isolda sp. & - & - & - & - & - & - & $*$ & - \\
\hline Plerolysippe sp. & - & - & - & - & $*$ & - & - & - \\
\hline Nereidae & * & $*$ & - & - & $*$ & $*$ & $*$ & - \\
\hline \multicolumn{9}{|l|}{ Oligochaeta } \\
\hline Peloscolex sp & - & $*$ & * & $*$ & $*$ & * & - & - \\
\hline Naididae & - & - & - & - & - & - & - & * \\
\hline Oligochaeta s/I & $*$ & - & $*$ & * & $*$ & * & $*$ & * \\
\hline Hirudinea & - & - & - & - & * & - & - & - \\
\hline
\end{tabular}

(\#) Conforme Trivinho-Strixino \& Strixino (1995) 
Densidade (número de indivíduos $/ 300 \mathrm{~cm}^{3}$ )

Est. 1

Est. 2
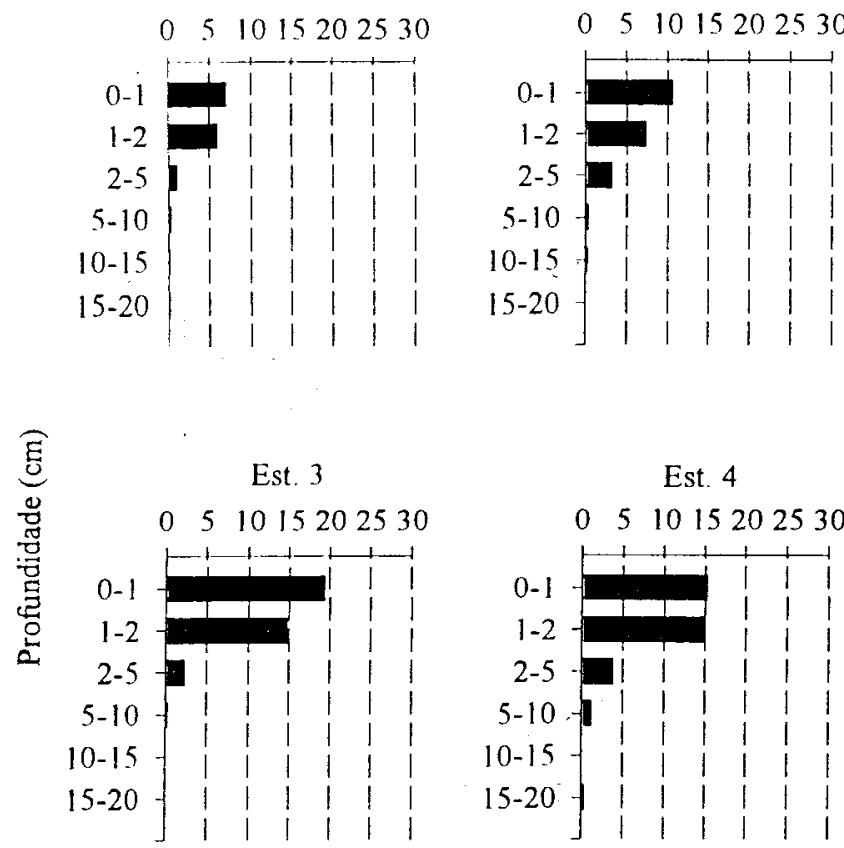

Est. 4

Est. 5
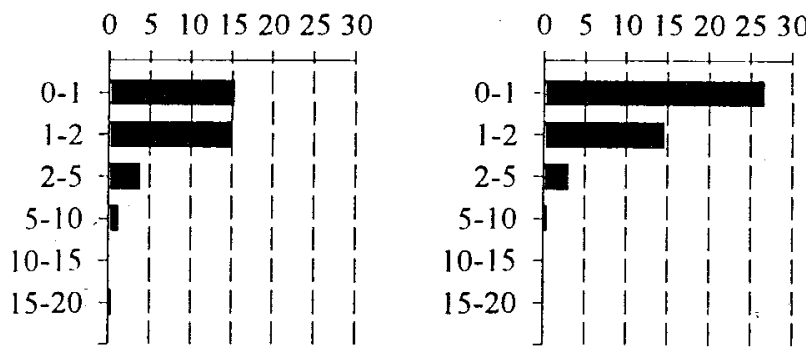

Est. 6

Est. 7

$\begin{array}{lllllll}0 & 5 & 1015202530\end{array}$
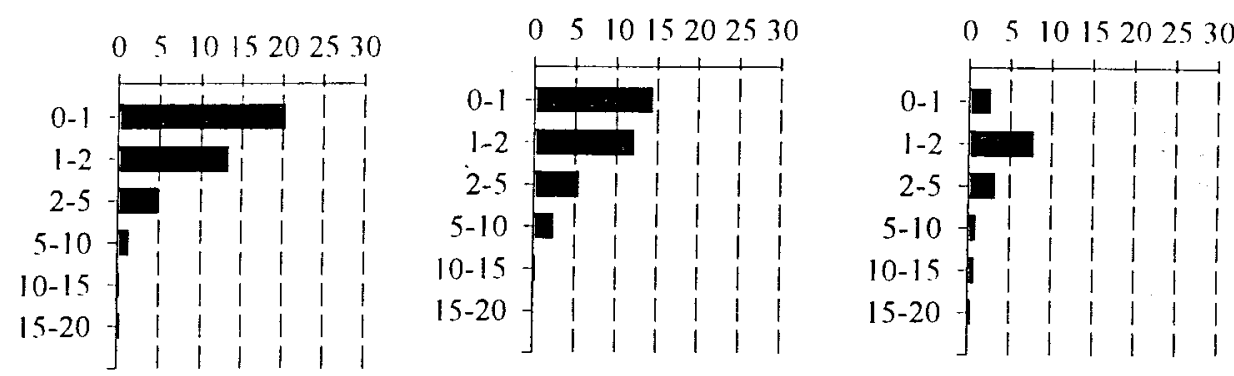

Fig. 4. Perfil vertical da densidade (ind. $/ 300 \mathrm{~cm}^{3}$ ) de organismos macrozoobentônicos encontrados na coluna de sedimento, nas estações de amostragem da região estuarina de Cananéia. (SP).

A distribuição das espécies na coluna vertical dos sedimentos sofreu mudanças a nível temporal que podem estar relacionadas tanto com as características físicas e químicas da água e do sedimento, quanto com as características hidrológicas e climatológicas da região. A penetração de oxigênio nos estratos mais profundos da coluna vertical do sedimento, também pode estar favorecendo o aumento na profundidade de enterramento das espécies, conjuntamente com a diminuição da concentração de MO, como ocorreu nas estações 6 e 7. A localização da RPD nestas estações está indicando a presença de oxigênio quase na totalidade da coluna de sedimentos.

Através de análise de correlação foram relacionados os valores de densidade de indivíduos na coluna dos sedimentos com os valores de potencial de óxido-redução (Tab. 2).
$\mathrm{Na}$ maioria das estações e períodos de amostragem, a relação entre o $\mathrm{Eh}$ e densidade de organismos foi significativa. Supõe-se que variações na distribuição das espécies e a densidade de organismos nos diferentes estratos sejam dependentes não só da presença de sedimentos oxidados, mas também de outros fatores como a disponibilidade de alimentos, relações interespecíficas, e respostas adaptativas à ação de predadores (Hines \& Comptois, 1985).

$\mathrm{Na}$ região de Cananéia a maioria dos organismos distribuiu-se entre $o$ sedimento subsuperficial e os primeiros $5 \mathrm{~cm}$ de profundidade na coluna vertical de sedimentos e a profundidade de enterramento aumentou nas áreas de maior salinidade (estação 6), fato este também observado por Dauer et al. (1987) para a Baía de Chesapeake (EUA). 
Tabela 2. Valores de correlação obtidos entre a densidade de organismos no perfil vertical dos sedimentos e os valores de óxido-redução, da região estuarina de Cananéia (SP) $\left(n=6,{ }^{* *} p<0,05 ;{ }^{*} p<0,5\right)$

\begin{tabular}{|l|l|l|l|l|l|l|l|l|}
\hline Mês/Estação & \multicolumn{1}{|c|}{$\mathbf{1}$} & $\mathbf{2}$ & $\mathbf{3}$ & $\mathbf{4}$ & $\mathbf{5}$ & $\mathbf{6}$ & \multicolumn{1}{|c|}{$\mathbf{7}$} & $\mathbf{8}$ \\
\hline Feverciro & $0,67^{*}$ & $0,97^{* *}$ & 0,48 & $0,96^{* *}$ & $0,67^{*}$ & $0,88^{* *}$ & 0,49 & 0,46 \\
\hline Maio & $0,97^{* *}$ & $0,66^{*}$ & $0,89^{* *}$ & 0,25 & 0,50 & $0,99^{* *}$ & $0,56^{*}$ & $0,59^{*}$ \\
\hline Agosto & $0,95^{* *}$ & $0,73^{*}$ & $0,59^{*}$ & $0,78^{*}$ & $0,76^{*}$ & 0,26 & $0,60^{*}$ & $0,83^{* *}$ \\
\hline Novembro & $0,57^{*}$ & $0,80^{*}$ & $0,97^{* *}$ & $0,84^{* *}$ & $0,93^{* *}$ & $0,73^{*}$ & $0,82^{* *}$ & $0,59^{*}$ \\
\hline
\end{tabular}

\section{Agradecimentos}

Ao CNPq pela concessão da bolsa de estudos (processo: 141579/94-3). Ao Instituto Oceanográfico-USP pelas facilidades oferecidas quanto ao uso da Base de Pesquisa "Dr. João de Paiva Carvalho"Cananéia, assim como a todo o pessoal da Base pela colaboração na obtenção das amostras. Ao Prof. Dr. José Galizia Tundisi pela orientação na elaboração deste trabalho.

\section{Referências bibliográficas}

Dauer, D. M.; Ewing, R. M. \& Rodi Jr., A. J. 1987. Macrobenthic distribution within the sediment along an estuarine salinity gradient. Int. Revue ges Hydrobiol., 72(5):529-538.

Duplisea, D. E \& Hargrave, B. T. 1996. Response of meiobenthic size-structure, biomass and respiration to sediment organic enrichment. Hydrobiologia, 339(1/3):161-170.

Flach, E. \& Heip, C. 1996. Vertical distribution of macrozoobenthos within the sediment on the continental slope of the Cogan Spur area (NE Atlantic). Mar. Ecol. Prog. Ser., 141(1/3):5566.

Flint, R. W. \& Kalke, R. D. $1986 . \quad$ Biological enhancement of estuarine benthic community structure. Mar. Ecol. Prog. Ser., 31(1):23-33.

Grizzle, R. E. \& Penniman, C. A. 1991. Effects of organic enrichment on estuarine macrofaunal benthos: a comparison of sediment profile imaging and traditional methods. Mar. Ecol. Prog. Ser., 74(2/3):249-262.

Hakanson, L. \& Jansson, M. 1983. Principles of lake sedimentodology. Berlim, Springer-Verlag. $316 \mathrm{p}$.

Hayes, F. R.; Reid, B. L. \& Cameron, M. L. 1958. Lake water and sediment. 11 Oxidatio-reduction relations at the mud-water interface. Limnol. Oceanogr., 3(3):308-317.
Hines, A. H. \& Comptois, K. L. 1985. Vertical distribution of infauna in sediments of a subestuary of central Chesapeake Bay. Estuaries, 8(3): 296-304.

Kirchner, W. B. 1975 . The effect of oxidized material on the vertical distribution of freshwater benthic fauna. Freswat. Biol., 5:423-429.

Nalepa, T. F. \& Robertson, A. 1981. Vertical distribution of the zoobenthos in Southeastern Lake Michigan with evidence of seasonal variation. Freshwat. Biol., 11:87-96.

Nilsson, H. C. \& Rosenberg, R. 1994. Hypoxic response of two marine benthic communities. Mar. Ecol. Prog. Ser., 115(3):209-217.

Rhoads, D. C. 1974. Organism-sediment relations on the muddy sea floor. Oceanogr. mar. Biol. Ann. Rev., 12:263-300.

Rhoads, D. C. \& Young. D. K. 1970. The influence of deposit-feeding organisms on sediments stability and community trophic structure. J. mar. Res., 28(2):150-178.

Schaeffer-Novelli, Y.; Mesquita, H. de S. L. \& Cintron-Molero, G. 1990. The Cananéia Lagoon estuarine system, São Paulo, Brazil. Estuaries, 13(2):193-203.

Trivinho-Strixino, S. \& Strixino, G. 1995. Larvas de Chironomidae (Diptera) do Estado de São Paulo. Guia de identificação e diagnose dos gêneros. São Carlos, PPG-ERN/UFSCAr. 229p.

Zobell, C. E. 1946. Studies on redox potential of marine sediments. Bull. Am. Ass. Petrol. Geol., 30(4):477-513.

(Manuscrito recebido 05 maio 1998; revisado 28 agosto 1998; aceito 18 março 1999) 